\title{
BMJ Open Decision-making impairment in patients with multiple sclerosis: a case-control study
}

\author{
Mauricio F Farez, Lucía Crivelli, Ramón Leiguarda, Jorge Correale
}

To cite: Farez MF, Crivelli L, Leiguarda $\mathrm{R}$, et al. Decisionmaking impairment in patients with multiple sclerosis: a case-control study. BMJ Open 2014;4: e004918. doi:10.1136/ bmjopen-2014-004918

- Prepublication history for this paper is available online. visit the journal online (http://dx.doi.org/10.1136/ bmjopen-2014-004918).

MFF and LC contributed equally.

Received 27 January 2014 Revised 6 June 2014 Accepted 10 June 2014 To view these files please

\section{ABSTRACT \\ Objectives: To assess decision-making under explicit risk conditions in relapsing-remitting multiple sclerosis patients and its relationship to decisions made under conditions of ambiguity. To assess cognitive functions related to decision-making performance in patients with multiple sclerosis (MS).}

Setting: MS center in Buenos Aires, Argentina. Participants: 27 patients with relapsing-remitting multiple sclerosis and 27 sex-matched, age-matched and education-matched healthy controls.

Intervention: Neuropsychological assessment and decision-making evaluation using the Game of Dice Task and the lowa Gambling Task.

Outcomes: Game of Dice Task and the lowa Gambling Task scores.

Results: Patients with MS showed significantly poorer performance on the Game of Dice Task, choosing disadvantageous dice more often ( $p=0.019)$, as well as significantly lower overall scores in the lowa Gambling Task ( $p=0.007$ ). Block analysis showed that patients with MS and controls had scores that were comparable for blocks 1 and 2 ( $p=0.15$ and $p=0.24$, respectively). Conversely, patients with MS scored poorly on blocks $4(p=0.003)$ and $5(p=0.023)$, the last two of the test, corresponding to decision-making under conditions of risk. Finally, the Game of Dice Task performance together with the last three blocks of the lowa Gambling Task were correlated with visuospatial learning, processing speed and working memory but not with executive functioning.

Conclusions: Patients with MS showed deficits in decision-making under risk conditions, which might be related to deficits in visuospatial learning, processing speed and working memory.

\section{INTRODUCTION}

Department of Neurology, Raúl Carrea Institute for Neurological Research (FLENI), Buenos Aires, Argentina

Correspondence to Dr Mauricio F Farez; mauriciofarez@gmail.com
Multiple sclerosis (MS) is an inflammatory demyelinating disease of the central nervous system that affects white matter, predominantly in the form of demyelinated plaques or lesions. ${ }^{1}$ It is nowadays being recognised more and more that, in addition to white matter damage, grey matter involvement also plays an important role in MS symptomatology and

\section{Strengths and limitations of this study}

- Patients with multiple sclerosis (MS) showed significantly poorer performance on the Game of Dice Task and the lowa Gambling Task. This may be related to deficits in visuospatial learning, processing speed and working memory.

- Disturbances in decision-making (DM) may affect quality of life as well as social environment interaction. The detection and characterisation of cognitive deficits in MS will therefore both help patients and improve rehabilitation therapies for these cases.

- The limitations of this study include the unblinded observational design and small cohort size; our findings need further validation in a larger cohort. Moreover, association is not causation, and the correlations observed between working memory, speed processing and DM may reflect associations between domains that are equally affected by an unmeasured and unrelated cognitive deficit.

progression. $^{2}$ Although some areas of the brain are affected more often than others, lesional distribution is thought to be random, with symptoms reflecting affected areas and patients presenting sensory, motor or cognitive disturbances. ${ }^{1} \mathrm{Up}$ to $70 \%$ of patients with MS may present cognitive disturbances at some point during disease development. ${ }^{3}$ Cognitive areas affected in MS include executive functioning, ${ }^{4}$ attention, ${ }^{5}$ information processing ${ }^{6}$ and memory, ${ }^{3}$ of which processing speed and visual memory are most commonly affected (52 and 54\%, respectively). ${ }^{37}$ Information processing efficiency is measured both by the brain's capacity to manipulate information during short periods (working memory) and by the time required to access necessary information for the task undertaken (processing speed). Impairment in processing speed can also affect other domains, such as executive function or working memory. ${ }^{8}$ Interestingly, increased demand for working memory is also associated with decreased processing speed. ${ }^{19}$ 
The Paced Auditory Serial Addition (PASAT) test, which measures working memory with high processing speed requirements, is one of the most sensitive tests available to detect cognitive impairment in patients with MS and it shows good correlation with deficit progression. ${ }^{2} 10$

As mentioned, impairment in the executive capabilities needed to successfully complete complex tasks and to adapt to shifting environments or demands has also been described in patients with MS, but less frequently than processing speed or visual memory. ${ }^{1}$

Regardless of impairment frequency, both executive function and working memory are key components in decision-making (DM) ${ }^{11-13} \mathrm{DM}$, the ability to choose between different options, is a key component of everyday functioning. When altered, it can have important negative consequences for the affected individuals, both socially and personally. ${ }^{14}$

DM can be assessed using the Game of Dice Task (GDT), which evaluates decisions made under explicit risk conditions in a task providing specific probabilities for positive and negative outcomes and foreseeable gains or losses resulting from each decision. ${ }^{15}{ }^{16}$ In this test, individuals have to guess, during 18 throws of a single dice, which number will appear, choosing either one, two, three or four numbers. Safe options are threeor four-number combinations, whereas single- or double-number combinations are considered risky. Executive function plays an important role in categorising and handling winning probabilities during the GDT. As previous studies have shown in healthy controls, GDT performance correlates with executive function as measured by the Modified Card Sorting Test (MCST) and individuals with reduced executive functioning take disadvantageous decisions in explicit risk situations. ${ }^{17}$

Feedback processing has also been shown to influence performance since individuals with impaired executive function can still perform adequately by learning from feedback provided after each throw. ${ }^{17}$ Finally, visual learning cues and working memory are other important factors that influence DM performance, and response to feedback or DM processing sometimes relies on the use of these capabilities. ${ }^{14}$ The relationship between DM and working memory was demonstrated by Bechara and colleagues by analysing participants with brain lesions in different locations and the impact on working memory and DM. Individuals having lesions affecting working memory have also poorer performance in DM tasks. ${ }^{11}$ In addition to this, working memory impairment underlies at least partly the deficits observed in DM under risk conditions in individuals with substance addiction. ${ }^{12}$

This study investigates DM under explicit risk conditions as measured by the GDT, a task previously tested in healthy controls, ${ }^{14}$ as well as under several disease conditions, such as Alzheimer's ${ }^{18}$ and Parkinson's. ${ }^{19}$ The main goal was to investigate the correlation between DM capabilities, executive function and working memory in patients with MS. An additional objective was to assess performance in GDT and compare the results to performance in the Iowa Gambling Task (IGT), a test evaluating learning and DM under ambiguous conditions, where risk is not explicitly explained to the individual, and learning which options are more advantageous is required. ${ }^{20}$ Briefly, participants have to choose among four decks of cards in 100 trials and win or lose certain amounts of fictitious money. Two decks are disadvantageous in the long term, as they are associated with immediate high gain but higher unpredictable future losses, and two decks are advantageous in the long term because they provide low immediate gain but lower unpredictable future losses. Since these contingencies are not explained to the participants, they have to learn to choose among the more advantageous decks.

DM under ambiguous conditions-as evaluated by the IGT-has been reported to be affected in many conditions such as Parkinson's disease, ${ }^{19}$ substance abuse, ${ }^{21}$ pathological gambling, ${ }^{22}$ anorexia nervosa ${ }^{23}$ and MS. ${ }^{24-27}$ In the latter, several studies have reported impaired DM under ambiguous conditions even in the absence of major mood, cognitive or physical impairment. Moreover, Simioni and colleagues showed that a decline in DM capacity under ambiguity can appear as an early deficit in patients with MS, and when retested after 2 years, individuals can show significant deterioration in IGT scores independently of other markers of cognitive, emotional or disability worsening. ${ }^{27}$ The study by Nagy and colleagues also reports that DM impairment in patients with MS can appear as an isolated deficit without the presence of generalised cognitive impairment and that this deficit is unrelated to executive function, as they did not detect a significant correlation between IGT scores and the Wisconsin Card Sorting Test. ${ }^{25}$

However, it remains unclear whether patients with MS show impairment when executing tasks with explicit rules that require strategy and feedback processing, nor is it clear whether DM performance under risk conditions is correlated with feedback processing, working memory and information processing speed. We hypothesise that DM under explicit risk conditions is altered in patients with MS (ie, they favour riskier option choices), in relationship with executive and/or memory impairment, and we tested for a correlation between these domains and DM capabilities in the study patients.

\section{METHODS \\ Patients}

Patients were recruited from the MS clinic at the Raúl Carrea Institute for Neurological Research (FLENI) by attending neurologists. Twenty-seven relapsing-remitting patients with MS fulfilling the $2005 \mathrm{McDonald}$ criteria ${ }^{28}$ with less than 2 years of disease duration and scores $<2$ on the Expanded Disability Status Scale (EDSS) underwent a complete neuropsychological evaluation. Patients presenting upper limb impairment, visual acuity or visual field deficits, a personal history of alcohol or drug 
abuse, head trauma, major psychiatric disorders or other neurological disorders or systemic illnesses were excluded from the study. All tests were conducted at least 90 days after full recovery from the most recent relapse or the discontinuation of steroid treatment. Twenty-seven participants matched for age, gender and educational level served as the controls. The study protocol was approved by the hospital Ethics Committee and all participants signed an informed consent form.

\section{Neuropsychological evaluation}

All participants completed Rao's Brief Repeatable Battery (BRB). ${ }^{29}$ This battery includes tests for the assessment of cognitive components typically affected in MS such as processing speed (digit symbol test), working memory (PASAT), verbal memory (selective reminding test, SRT), visuospatial learning (7/24 spatial recall test) and verbal fluency (word list generation). Executive functions were studied using the MCST, ${ }^{30}$ and depression was assessed using the Fast Screen version of the Beck Depression Inventory. ${ }^{31}$

\section{Game of Dice Task}

The GDT is used to evaluate DM in a gambling situation under explicit rules ${ }^{16}$ because rules for gains and losses as well as success probabilities are explicit and stable. Participants have to guess, during 18 throws of a single dice, which number will appear before selecting a single number (probability of winning $\$ 1000$ was $1: 6=16.7 \%$ ) or a combination of two (probability of winning $\$ 500$ was 2:6=33.3\%), three (probability of winning $\$ 200$ was $3: 6=50 \%$ ) or four numbers (probability of winning $\$ 100$ was $4: 6=66.6 \%$ ). Safe options are three-number or fournumber combinations, whereas single-number or double-number combinations are considered risky. The participants were aware of the duration of the test, affording the application of a long-term strategy to increase the outcome. The GDT net score was calculated by subtracting the number of risky selections from the number of safe selections. Additionally, selection proportions were also calculated for each round. Finally, feedback processing was assessed to determine whether receiving negative feedback influenced future throws.

\section{lowa Gambling Task}

The IGT was designed to simulate real-life DM under ambiguity; this means that the outcome probabilities are unknown. The task consists of choosing a card from one of four decks: decks A and B are disadvantageous in the long term, as they are associated with immediate high gain but higher unpredictable future losses, whereas decks $\mathrm{C}$ and $\mathrm{D}$ are advantageous in the long term because they provide low immediate gain but lower unpredictable future losses. Moreover, the disadvantageous decks differ from each other since losses are more frequent but of a lower value for deck A than for deck $\mathrm{B}$, which, in contrast, is associated with higher gains but overall higher losses than deck A. Likewise, among the advantageous decks, losses are more frequent for deck $\mathrm{C}$ but of a lower value than for deck $\mathrm{D}$, which is associated with higher overall gain. The participants were unaware of gain/loss probabilities when beginning the task. It was expected that, over the course of 100 card selections, optimal performers would progressively develop the strategy of choosing more often from advantageous decks. The IGT net score was calculated by subtracting the number of disadvantageous deck selections from the number of advantageous deck selections. Additionally, all 100-card selections were subdivided into five blocks of 20 selections each, and scores for these blocks were also calculated. It has been argued that the first two IGT blocks are those that actually measure DM under ambiguity, as, once participants learn which decks are more disadvantageous, decisions are made under risk conditions (blocks 3-5). ${ }^{13}{ }^{14}$ Blocks 1 and 2 combined were considered the ambiguous IGT score block, and the former blocks 3-5 were combined and considered to be explicit-risk blocks.

\section{Statistical analysis}

Unless otherwise mentioned, the data are expressed as the mean \pm SEM. Normal distribution was assessed using a Kurtosis test; if variables were not normally distributed, a non-parametric Mann-Whitney test was applied. Otherwise, a t test was performed to evaluate the differences between patients with MS and controls. Bonferroni correction was applied to adjust for multiple testing on the BRB tests, and $p$ values $\leq 0.004$ were considered significant.

Changes during GDT performance were assessed by applying a proportional odds regression model. For feedback analysis, participants were considered to respond to feedback when they switched to an advantageous option after selecting a disadvantageous one and losing money. The proportion of participants switching to an advantageous option after receiving negative feedback was analysed by applying a mixed-effects logistic regression model.

Forward stepwise linear regression analysis was used to determine which cognitive areas correlated with GDT performance. Univariate analysis was performed for each test included in the BRB and the MCST; tests with $p$ values $<0.15$ were ranked and kept for the next step. We then added as a first variable the one having the highest correlation with the dependent variable (GDT score) and, if it was significant, we continued with the next variable until no more were available. Partial $\mathrm{F}$ tests were conducted at every step, and non-significant variables were removed.

To construct a longitudinal model able to detect IGT changes during the five blocks of the test, a mixed-effects regression approach was used. The model included the IGT score as the dependent variable and diagnosis, block, and interaction as independent variables. The systematic part of the model included diagnosis and block-specific intercepts and slopes and, for the 
Table 1 Participants' clinical characteristics and demographics

\begin{tabular}{lll}
\hline & $\begin{array}{l}\text { Healthy } \\
\text { controls } \\
(\mathbf{n}=\mathbf{2 7})\end{array}$ & $\begin{array}{l}\text { Patients } \\
\text { with } \\
\text { MS }(\mathbf{n}=\mathbf{2 7})\end{array}$ \\
\hline Age (years, mean \pm SD) & $\begin{array}{l}33.86 \pm 13.51 \\
10: 17\end{array}$ & $\begin{array}{l}33.3 \pm 9.7 \\
10: 17\end{array}$ \\
Gender (M:F) & $14.06 \pm 2.3$ & $15.33 \pm 2.21$ \\
Education (years, mean \pm SD) & NA & $7.9 \pm 8.1$ \\
$\begin{array}{l}\text { Duration of disease (months, } \\
\text { mean } \pm S D)\end{array}$ & NA & $1.03 \pm 0.80$ \\
\hline EDSS (mean $\pm S D)$ & & \\
\hline EDSS, Expanded Disability Status Scale. &
\end{tabular}

random analysis, a random shift in the intercept and slope was added for each participant. $\mathrm{p}$ Values below 0.05 (two-tailed) were considered significant. Mediational analyses were performed using the Preacher and Hayes bootstrapped test of mediation. All statistical analyses were performed using Stata V.12 (Statacorp LP, Texas, USA).

\section{RESULTS}

Clinical, demographic and neuropsychological characteristics of study participants

Clinical and demographic characteristics of the study participants are presented in table 1 . The results of the $\mathrm{BRB}$ as well as of other tests performed by patients and healthy controls (HC) are reported in table 2. Patients with MS showed deficits in selective reminding tests, the Spatial Recall Test and the PASAT, but, after Bonferroni correction for multiple testing, none of the differences was statistically significant.

\section{DM under risk is impaired in patients with MS}

Results of DM assessment are presented in table 3 . During the GDT, patients with MS made disadvantageous choices (single and pair dice) more frequently than $\mathrm{HC}$ $(29.9 \% \pm 5.6$ vs $14.4 \% \pm 2.4, \mathrm{p}=0.019$; see figure $1 \mathrm{~A})$.

These deficits could be caused by alterations in the ability to incorporate feedback (patients fail to learn after losing money using disadvantageous dice) or by impairment in executive functions (patients fail to elaborate the right decisions correctly).

If participants were able to successfully use feedback received during task execution, the frequency of advantageous dice choice would be expected to increase progressively over rounds. To assess whether this was the case, we tested whether the proportion of each of the four decisions shifted with successive rounds and whether this change differed between patients and controls. No significant use of feedback was detected as the rounds progressed (OR 1.03, 95\% CI 0.99 to 1.06 ), nor was there significant interaction between rounds and diagnosis (OR 1.03, 95\% CI 0.98 to 1.07). Thus, neither patients nor controls modified choices significantly during the task (see figure 1B). Owing to the fact that some individuals never chose a disadvantageous option or, if they did, they may still not have received negative feedback, we further investigated whether choosing the disadvantageous dice and getting negative feedback (ie, losing money) influenced the shift to an advantageous option in either group. No significant difference in the proportion of individuals using negative feedback to modify decisions was observed ( $36 \%$ for healthy controls vs $33 \%$ in patients with MS, $\mathrm{p}=0.48)$. Thus, deficits in DM under risk observed in patients with MS did not seem to be caused by deficiencies in feedback processing.

\section{Effect of visuospatial learning, processing speed and working memory on DM under risk}

Since feedback processing deficit was not the reason for the underlying DM deficits observed in patients with MS and since it has been proposed that decisions made under explicit risk are correlated with executive functioning and memory, ${ }^{12} 32$ we also investigated whether

Table 2 Neuropsychological assessment

\begin{tabular}{llll}
\hline & Healthy controls $(\mathbf{n = 2 7})$ & Patients with MS (n=27) & $\mathbf{p}$ Value \\
\hline PASAT 2.0 s & $47.63 \pm 1.75$ & $42.19 \pm 1.96$ & 0.043 \\
PASAT 3.0 s & $41.52 \pm 2.04$ & $34.85 \pm 2.44$ & 0.041 \\
Symbol Digit Modalities Test & $61.15 \pm 2$ & $55.78 \pm 2.48$ & 0.10 \\
SRT-Total Learning & $48.55 \pm 2.07$ & $44.41 \pm 2.93$ & 0.25 \\
SRT-Long-term storage & $40.89 \pm 2.17$ & $35.33 \pm 3.14$ & 0.15 \\
SRT-Delayed Recall & $9.33 \pm 0.30$ & $8 \pm 0.42$ & 0.012 \\
7/24 Spatial Recall Task-Total Recall & $30.82 \pm 0.68$ & $29.59 \pm 0.76$ & 0.24 \\
7/24 Spatial Recall Task-Delayed Recall & $6.22 \pm 0.22$ & $5.33 \pm 0.41$ & 0.06 \\
Word list generation & $21.74 \pm 1.59$ & $18.52 \pm 0.62$ & 0.023 \\
MCST-Categories & $5.96 \pm 0.03$ & $5.81 \pm 0.15$ & $0.68 \pm 0.36$ \\
MCST-Perseverative errors & $0.48 \pm 0.31$ & $3.8 \pm 1.25$ & 0.54 \\
MCST-Non-perseverative errors & $3 \pm 1.12$ & $3.0 \pm 2.6$ & 0.68 \\
BDI-FS (mean \pm SD) & $2.6 \pm 2.9$ & 0.55 \\
\hline BDI-FS, Beck Depression Inventory-Fast Screen; PASAT, Paced Auditory Serial Addition; MCST, Modified Card Sorting Test; SRT, selective \\
reminding test. & &
\end{tabular}


Table 3 Decision-making assessment

\begin{tabular}{|c|c|c|c|}
\hline & $\begin{array}{l}\text { Healthy controls } \\
\text { (mean } \pm \text { SEM) }\end{array}$ & $\begin{array}{l}\text { Patients with MS } \\
\text { (mean } \pm \text { SEM) }\end{array}$ & p Value \\
\hline \multicolumn{4}{|c|}{ Assessment of decisions under risk (GDT results) } \\
\hline Frequency of disadvantageous choices & $14.4 \% \pm 2.4 \%$ & $29.9 \% \pm 5.6 \%$ & 0.019 \\
\hline Money balance at the end of the game* & $500 \pm 1991$ & $-100 \pm 3007$ & 0.45 \\
\hline \multicolumn{4}{|c|}{ Assessment of decisions under ambiguity (IGT results) } \\
\hline Total IGT score $(\mathrm{C}+\mathrm{D})-(\mathrm{A}+\mathrm{B})$ & $32.9 \pm 4.1$ & $14.9 \pm 4.9$ & 0.007 \\
\hline IGT score Block 1 & $-1.04 \pm 0.94$ & $-2.82 \pm 0.79$ & 0.15 \\
\hline IGT score Block 2 & $3.56 \pm 1.16$ & $1.78 \pm 0.94$ & 0.24 \\
\hline IGT score Block 3 & $7.78 \pm 1.15$ & $4.81 \pm 1.15$ & 0.08 \\
\hline IGT score Block 4 & $11.63 \pm 1.3$ & $5.70 \pm 1.4$ & 0.003 \\
\hline IGT score Block 5 & $10.97 \pm 1.5$ & $5.41 \pm 1.8$ & 0.023 \\
\hline
\end{tabular}

an association existed between these domains and GDT performance.

No significant correlation was detected between executive function-as measured by the MCST - and DM in patients with MS $(p=0.29)$. We then evaluated whether working and visual memory-two traits frequently affected in MS-correlated with DM abilities. A significant correlation was detected between disadvantageous choices and both the $7 / 24$ Spatial Recall Test (a test of visuospatial memory, $\mathrm{p}=0.021$ ) and PASAT $2^{\prime \prime}$
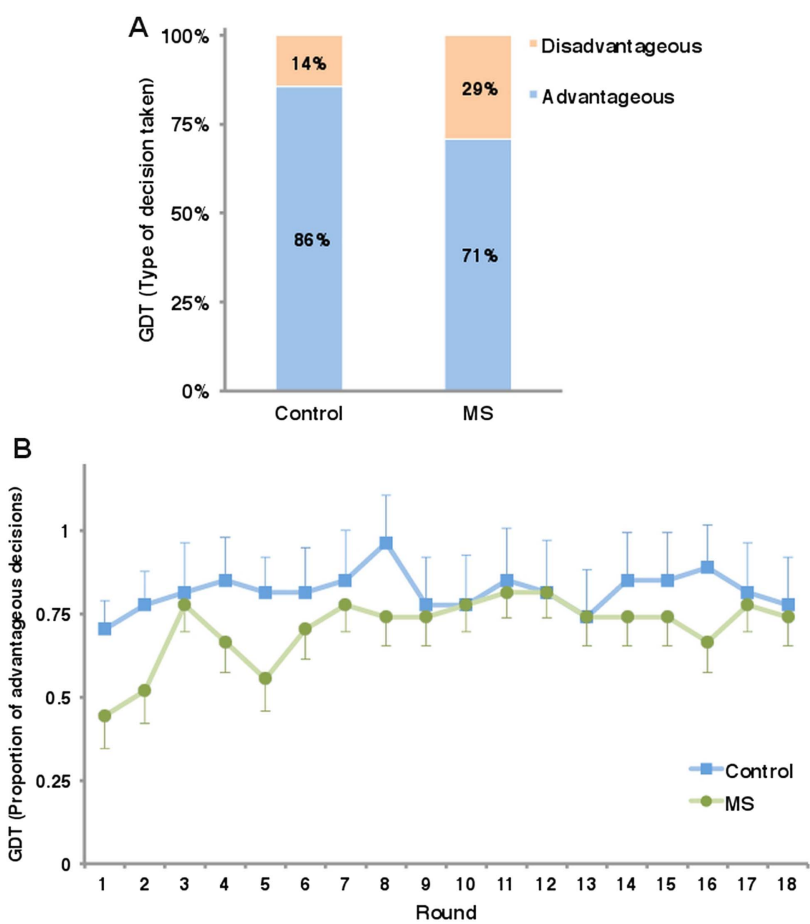

Figure 1 Game of dice task results. (A) Total percentage of advantageous or disadvantageous choices for patients with multiple sclerosis (MS) and healthy controls. Choosing one or two dice was considered a disadvantageous choice. See table 3 for SEM and associated $p$ values. (B) Proportion of advantageous decisions for patients with MS and healthy controls for each of the 18 rounds. Mean \pm SEM is shown. performance (a working memory test requiring a high processing speed, $\mathrm{p}=0.025)$. As performance on both tests decreased, participants tended to choose higher risk options in the GDT. Thus, DM performance under risk seems, in patients with MS, to be related to visuospatial learning and working memory/speed processing but not to executive function. Finally, we assessed whether the effect of visual memory on DM is mediated by working memory and we found that the relationship between visual memory and DM was partially mediated by working memory $(27 \%)$ and that the direct effect remained significant $(p=0.01)$. Thus, although some of the effect of visuospatial memory is mediated by working memory, the majority of the effect is direct.

DM assessed by IGT in patients with MS is affected only in blocks involving decisions made under risk

IGT differs from GDT essentially in that outcome probabilities are unknown and participants have to learn which options are advantageous. Throughout the 100 test trials, patients with MS chose less advantageous decks significantly more often, as reflected by lower total IGT scores $(32.9 \pm 4.1$ for $\mathrm{HC}$ vs $14.9 \pm 4.9$ for patients with MS, $\mathrm{p}=0.007$; see figure $2 \mathrm{~A}$ ). It has been argued that IGT actually assesses DM in a dual fashion. ${ }^{13}$ The first two blocks truly correspond to decisions made under ambiguity, as individuals follow their guesses and hunches to choose between decks. However, once participants figure out which decks are associated with worse outcomes, they learn to choose the better decks so that, for the last three blocks, decisions are made based on relatively known risks, ${ }^{13}{ }^{33}$ in which executive control is thought to play a more important role. ${ }^{14}$

We then assessed whether IGT scores changed over blocks (meaning that participants learned to avoid disadvantageous decks over time and perform better) and whether the slope of this change was different between patients and controls in each block. As shown in figure 2B and table 3, no significant differences were detected between patients and controls during the first two blocks ( $p=0.15$ and $p=0.24$ for blocks 1 and 2 , respectively). 


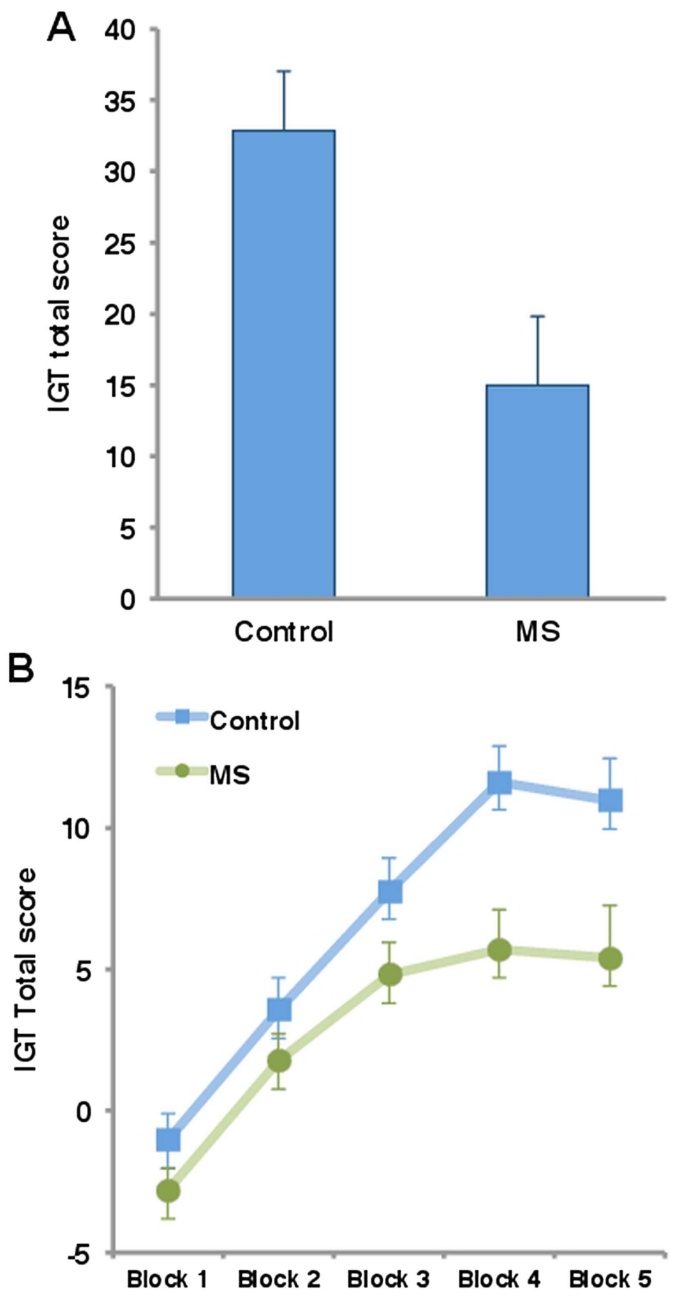

Figure 2 lowa gambling task results. (A) Total IGT scores $((C+D)-(A+B))$ for patients with $M S$ and healthy controls.

Mean \pm SEM is shown. $(B)$ Total IGT scores $((C+D)-(A+B))$ by block for patients with MS and healthy controls. Mean \pm SEM is shown.

Interestingly, starting from block 3 and reaching significance in blocks $4 \quad(\mathrm{p}=0.003)$ and $5 \quad(\mathrm{p}=0.023)$, patients with MS differed from HC, as they stopped progressing in their scores. Patients with MS responded normally when making decisions under purely ambiguous conditions but started failing once decisions had to be made under risky ones (not formally tested, assumed to be this way based on prior evidence).

We then hypothesised that impaired DM observed during the last IGT blocks responded to the same cause as impaired performance in the GDT and, therefore, that a correlation between scores as well as an association between visuospatial learning and working memory should be present. Indeed, GDT performance correlated positively to scores in the last three IGT blocks $(p=0.021$ ), and correlations between visuospatial learning and working memory performance were also found for the last three IGT blocks (risk blocks, $\mathrm{p}=0.05$ and $\mathrm{p}=0.023$, respectively), but not for the first two (ambiguous blocks, $\mathrm{p}=0.33$ and $\mathrm{p}=0.49$, respectively).

\section{DISCUSSION}

We observed impaired DM in patients with MS under stable and explicit rules for gain and loss. The study results also suggested that deficits correlated with visuospatial and working memory performance, but not with feedback processing or executive function.

Studies in primates ${ }^{34}$ as well as humans ${ }^{35}$ have shown that the dorsolateral prefrontal cortex is key in working memory function, as measured by a variety of tasks. The claim that working memory is necessary for DM comes from studies showing that individuals with lesions in this area and low performance in working memory are also affected in their DM capacity. ${ }^{11}$ Working memory deficits are also commonly observed in patients with MS. ${ }^{29}$ The nature of this particular deficit is beyond the scope of this manuscript, but the accumulation of white and grey matter lesions in a random fashion over time seems to be the most likely underlying mechanism. ${ }^{3}$ However, our results highlight the fact that memory deficits are not only important per se, but can also affect other equally or even more important areas, such as DM.

Even though visuospatial impairment has been consistently observed in patients with $\mathrm{MS},{ }^{29}{ }^{36}$ its relation to DM has not been addressed. Visuospatial memory is closely linked to working memory, and pre-existing deficits in visuospatial memory in patients with MS have been found to affect working memory function. ${ }^{36}$ Quantification of the contribution of visuospatial and working memory to DM in MS is an interesting question to be addressed in future studies and until then these findings should be interpreted with caution.

DM under ambiguous and risky conditions and the relationship between them were also studied. No significant deficit in ambiguous decisions was detected, as measured by the first two blocks of the IGT, whereas significant impairment in learning and performance in the last three blocks was observed. DM (as measured by the IGT) has been previously shown to be impaired in patients with MS, ${ }^{24-27} 37$ but no distinction has been made regarding the type of DM affected. Overall, previous studies have found poorer performance in later IGT blocks, with preserved DM during the initial blocks. ${ }^{24-27} 37$

Little is known about the natural history of DM disturbances in MS; as mentioned, the study population comprised mostly recently diagnosed patients with MS. One longitudinal study reassessing IGT scores at 2-year intervals showed that DM deficits could progress over time. ${ }^{27}$ Interestingly, IGT performance in primary progressive MS (PPMS) seems to be significantly affected compared to $\mathrm{HC}$ and secondary progressive MS (SPMS) ${ }^{38}$ highlighting the heterogeneous nature of DM impairments.

The limitations of this study include the unblinded observational design and small cohort size. Moreover, association is not causation, and the correlations observed between working memory, speed processing and DM may reflect associations between domains that are equally affected by an unmeasured and unrelated cognitive deficit. Several factors that could have affected 
DM performance of patients with MS, such as depression, physical impairment (eg, decreased visual acuity or motor dysfunction) or limited processing speed (the most common cognitive deficit in MS), were each addressed. ${ }^{39}$ Given the inclusion criteria applied, patients were mostly recently diagnosed and without physical handicaps. No significant differences in depression scale scores were observed, nor was physical disability an issue; all individuals had EDSS scores below or equal to 2. Finally, GDT is not a timed test; therefore, it is unlikely that processing speed affected patient performance. Nevertheless, our findings needs further validation in a larger cohort of patients with MS.

\section{CONCLUSIONS}

Our study shows specific DM deficits in patients with MS. Disturbances in DM may affect quality of $\operatorname{life}^{24}$ as well as social environment interaction. ${ }^{40}$ The detection and characterisation of cognitive deficits in MS will therefore both help patients and improve rehabilitation therapies for these cases.

Contributors MFF, LC, RL and JC were involved in conception and design, acquisition of data, or analysis and interpretation of data; (2) drafting the article or revising it critically for important intellectual content; and (3) final approval of the version to be published.

Funding This study was supported by funding from the Raúl Carrea Institute for Neurological Research (FLENI) and by Merck-Serono Argentina.

Competing interests MFF received honoraria and professional travel/ accommodations stipends from Merck-Serono Argentina and Novartis Argentina. JC is a board member of Merck-Serono Argentina, Novartis Argentina, Biogen-Idec LATAM and Merck-Serono LATAM. JC received reimbursement to develop educational presentations for Merck-Serono Argentina, Merck-Serono LATAM, Biogen-Idec Argentina, Novartis Argentina, Novartis LATAM, Genzyme Argentina and TEVA-Tuteur Argentina as well as professional travel/accommodation stipends.

Patient consent Obtained.

Ethics approval Ethics committee.

Provenance and peer review Not commissioned; externally peer reviewed.

Data sharing statement No additional data are available.

Open Access This is an Open Access article distributed in accordance with the Creative Commons Attribution Non Commercial (CC BY-NC 3.0) license, which permits others to distribute, remix, adapt, build upon this work noncommercially, and license their derivative works on different terms, provided the original work is properly cited and the use is non-commercial. See: http:// creativecommons.org/licenses/by-nc/3.0/

\section{REFERENCES}

1. Noseworthy $\mathrm{JH}$, Lucchinetti C, Rodriguez $\mathrm{M}$, et al. Multiple sclerosis. N Engl J Med 2000;343:938-52.

2. $\mathrm{B} ø \mathrm{~L}$. The histopathology of grey matter demyelination in multiple sclerosis. Acta Neurol Scand Suppl 2009(189):51-7.

3. Chiaravalloti ND, DeLuca J. Cognitive impairment in multiple sclerosis. Lancet Neurol 2008;7:1139-51.

4. Drew M, Tippett LJ, Starkey NJ, et al. Executive dysfunction and cognitive impairment in a large community-based sample with multiple sclerosis from New Zealand: a descriptive study. Arch Clin Neuropsychol 2008;23:1-19.

5. Crivelli L, Farez MF, González CD, et al. Alerting network dysfunction in early multiple sclerosis. $J$ Int Neuropsychol Soc 2012;18:757-63.
6. De Sonneville LMJ, Boringa JB, Reuling IEW, et al. Information processing characteristics in subtypes of multiple sclerosis. Neuropsychologia 2002;40:1751-65.

7. Benedict RHB, Cookfair D, Gavett R, et al. Validity of the minimal assessment of cognitive function in multiple sclerosis (MACFIMS). $J$ Int Neuropsychol Soc 2006;12:549-58.

8. Kalmar JH, Gaudino EA, Moore NB, et al. The relationship between cognitive deficits and everyday functional activities in multiple sclerosis. Neuropsychology 2008;22:442-9.

9. Lengenfelder J, Bryant D, Diamond BJ, et al. Processing speed interacts with working memory efficiency in multiple sclerosis. Arch Clin Neuropsychol 2006;21:229-38.

10. Fischer JS, Rudick RA, Cutter GR, et al. The multiple sclerosis functional composite measure (MSFC): an integrated approach to MS clinical outcome assessment. Mult Scler 1999;5:244-50.

11. Bechara A, Damasio H, Tranel D, et al. Dissociation of working memory from decision making within the human prefrontal cortex. J Neurosci 1998;18:428-37.

12. Bechara A, Martin EM. Impaired decision making related to working memory deficits in individuals with substance addictions. Neuropsychology 2004;18:152-62.

13. Brand M, Recknor EC, Grabenhorst F, et al. Decisions under ambiguity and decisions under risk: correlations with executive functions and comparisons of two different gambling tasks with implicit and explicit rules. J Clin Exp Neuropsychol 2007;29:86-99.

14. Brand M, Labudda K, Markowitsch HJ. Neuropsychological correlates of decision-making in ambiguous and risky situations. Neural Netw 2006;19:1266-76.

15. Bechara A, Tranel D, Damasio H. Characterization of the decision-making deficit of patients with ventromedial prefrontal cortex lesions. Brain 2000;123(Pt 11):2189-202.

16. Brand M, Fujiwara E, Borsutzky S, et al. Decision-making deficits of korsakoff patients in a new gambling task with explicit rules: associations with executive functions. Neuropsychology 2005;19:267-77.

17. Schiebener J, Zamarian L, Delazer M, et al. Executive functions, categorization of probabilities, and learning from feedback: what does really matter for decision making under explicit risk conditions? $J$ Clin Exp Neuropsychol 2011;33:1025-39.

18. Delazer M, Sinz H, Zamarian L, et al. Decision-making with explicit and stable rules in mild Alzheimer's disease. Neuropsychologia 2007;45:1632-41.

19. Rossi M, Gerschcovich ER, de Achaval D, et al. Decision-making in Parkinson's disease patients with and without pathological gambling Euro J Neurol 2010;17:97-102.

20. Bechara A, Damasio AR, Damasio H, et al. Insensitivity to future consequences following damage to human prefrontal cortex. Cognition 1994:50:7-15.

21. Bechara A, Damasio H. Decision-making and addiction (part I): impaired activation of somatic states in substance dependent individuals when pondering decisions with negative future consequences. Neuropsychologia 2002;40:1675-89.

22. Cavedini P, Riboldi G, Keller R, et al. Frontal lobe dysfunction in pathological gambling patients. Biol Psychiatry 2002;51:334-41.

23. Cavedini P, Bassi T, Ubbiali A, et al. Neuropsychological investigation of decision-making in anorexia nervosa. Psychiatry Res 2004;127:259-66.

24. Kleeberg J, Bruggimann L, Annoni J-M, et al. Altered decision-making in multiple sclerosis: a sign of impaired emotional reactivity? Ann Neurol 2004;56:787-95.

25. Nagy H, Bencsik K, Rajda C, et al. The effects of reward and punishment contingencies on decision-making in multiple sclerosis. $J$ Int Neuropsychol Soc 2006;12:559-65.

26. Roca M, Torralva T, Meli F, et al. Cognitive deficits in multiple sclerosis correlate with changes in fronto-subcortical tracts. Mult Scler 2008;14:364-9.

27. Simioni S, Ruffieux C, Kleeberg J, et al. Progressive decline of decision-making performances during multiple sclerosis. $J$ Int Neuropsychol Soc 2009;15:291-5.

28. Polman $\mathrm{CH}$, Reingold SC, Edan $\mathrm{G}$, et al. Diagnostic criteria for multiple sclerosis: 2005 revisions to the 'McDonald Criteria'. Ann Neurol 2005;58:840-6.

29. Rao SM, Leo GJ, Bernardin L, et al. Cognitive dysfunction in multiple sclerosis. I. Frequency, patterns, and prediction. Am Acad Neurol 1991;41:685-91.

30. Nelson HE. A modified card sorting test sensitive to frontal lobe defects. Cortex 1976;12:313-24.

31. Benedict RHB, Fishman I, McClellan MM, et al. Validity of the Beck Depression Inventory-fast screen in multiple sclerosis. Mult Scler 2003;9:393-6. 
32. Brand M, Labudda K, Kalbe E, et al. Decision-making impairments in patients with Parkinson's disease. Behav Neurol 2004;15:77-85.

33. Maia TV, McClelland JL. A reexamination of the evidence for the somatic marker hypothesis: what participants really know in the lowa gambling task. Proc Natl Acad Sci USA 2004;101:16075-80.

34. Goldman-Rakic PS. Working memory and the mind. Sci Am 1992;267:110-17.

35. Courtney SM, Ungerleider LG, Keil K, et al. Transient and sustained activity in a distributed neural system for human working memory. Nature 1997;386:608-11.

36. Foong J, Rozewicz L, Quaghebeur G, et al. Executive function in multiple sclerosis. The role of frontal lobe pathology. Brain 1997; 120(Pt 1):15-26.
37. García-Molina A, Rodríguez Rajo P, Vendrell Gómez P, et al. [Orbitofrontal dysfunction in multiple sclerosis: lowa Gambling Task]. Psicothema 2008;20:445-9.

38. García-Molina A, Enseñat-Cantallops A, Sánchez-Carrión R, et al. [Assessment of decision-making capacity in primary and secondary progressive multiple sclerosis]. Neurologia 2009;24:94-7.

39. DeLuca J, Chelune GJ, Tulsky DS, et al. Is speed of processing or working memory the primary information processing deficit in multiple sclerosis? J Clin Exp Neuropsychol 2004;26:

550-62.

40. Bar-On R, Tranel D, Denburg NL, et al. Exploring the neurological substrate of emotional and social intelligence. Brain 2003;126: 1790-800. 